\title{
Isoscalar Giant Quadrupole Resonance State in the Relativistic Approach with the Momentum-Dependent Self-Energies
}

\author{
Tomoyuki Maruyama ${ }^{1,2}$ and Satoshi $\mathrm{Chiba}^{3}$ \\ ${ }^{1}$ College of Bioresource Sciences \\ Nihon University \\ Fujisawa, Kanagawa 252-8510, Japan \\ 2 The Institute of Physical and Chemical Research (RIKEN) \\ Hirosawa 2-1, Wako-shi, Saitama 351-0198, Japan \\ 3 Advanced Science Research Center, \\ Japan Atomic Energy Research Institute, \\ Tokai, Ibaraki 319-1195, Japan
}

\begin{abstract}
We study the excited energy of the isoscalar giant quadrupole resonance with the scaling method in the relativistic many-body framework. In this calculation we introduce the momentum-dependent parts of the Dirac self-energies arising from the one-pion exchange on the assumption of the pseudo-vector coupling with nucleon field. It is shown that this momentumdependence enhances the Landau mass significantly and thus suppresses the quadrupole resonance energy even giving the small Dirac effective mass which causes a problem in the momentum-independent mean-field theory.
\end{abstract}


The past decades have seen many successes in the relativistic treatment of the nuclear many-body problem. The relativistic framework has big advantages in several aspects [1]: a useful Dirac phenomenology for the description of nucleon-nucleus scattering [2,3], the natural incorporation of the spin-orbit force [1] and the structure of extreme nuclei [4]. These results have shown that there are large attractive scalar and repulsive vector fields, and that the nucleon effective mass becomes small in the medium.

The small effective mass enlarges the Fermi velocity, and it causes troubles in some observables. For example, the isoscalar giant quadrupole resonance (ISGQR) state is predicted at a too high excitation energy due to the large Fermi velocity [5]. In this subject, it is assumed that the momentum-dependence of the Dirac fields is negligible in the low energy region, particularly below the Fermi level. In fact, only very small momentumdependence has appeared in the relativistic Hartree-Fock (RHF) calculation though the Fock contribution is not small [6,7]. Thus the Fock contributions are thought to be incorporated into the relativistic Hartree $(\mathrm{RH})$ approximation by introducing complicated density-dependent interaction and fitting parameters [4, 6, 8 ].

In the high energy region the vector-fields must become very small to explain the optical potential of the proton-nucleus elastic scattering [2,9] and the transverse flow in the heavy-ion collisions [10]. A Dirac-Bruckner-Hartree-Fock (DBHF) calculation has also shown that the momentum-dependence changes the nuclear equation of state noticeably [11]. Furthermore, Weber et al. [9] have suggested that the Fermi velocity does not correspond to the effective mass uniquely when introducing the momentum-dependence of the Dirac fields.

We can easily suppose that it is the one-pion exchange force which produces the major momentum-dependence because the interaction range is largest. In this paper, thus, we introduce the momentum-dependence to the Dirac fields due to the one-pion exchange, and discuss how the Fock parts given by the one-pion exchange affects the excitation energy of ISGQR. Actually we use the scaling method in the way of Ref. [5] which is proved to give consistent results with RPA for the giant multipole states in the non-relativistic framework [12]. This relation has been confirmed also in the relativistic framework for the monopole vibration mode [13].

Let us consider the infinite nuclear matter system with the isospin symmetry. The 
nucleon propagator in the self-energy $\Sigma$ is given by

$$
S^{-1}(p)=\not p-M-\Sigma(p)
$$

where $\Sigma(p)$ has a Lorentz scalar part $U_{s}(p)$ and a Lorentz vector part $U_{\mu}(p)$ as

$$
\Sigma(p)=-U_{s}(p)+\gamma^{\mu} U_{\mu}(p)
$$

For the future convenience we define the effective mass and the kinetic momentum as follows.

$$
\begin{gathered}
M^{*}(p)=M-U_{s}(p), \\
\Pi_{\mu}(p)=p_{\mu}-U_{\mu}(p) .
\end{gathered}
$$

Using the on-mass-shell condition that $\Pi^{2}(p)-M^{* 2}(p)=0$, the single particle energy with momentum $\boldsymbol{p}$ is defined as

$$
\varepsilon(\boldsymbol{p})=\left.p_{0}\right|_{\text {on-mass-shell }}=\sqrt{\Pi^{2}(\boldsymbol{p})+M^{* 2}(\boldsymbol{p})}+U_{0}(\boldsymbol{p}) .
$$

Next we consider the variation of the total energy in the quadrupole deformation in order to discuss ISGQR with the scaling method. Using the scaling method, first, we vary the density-distribution from the normal nuclear matter distribution $\tilde{\rho}_{0}(\mathbf{r})$ as

$$
\tilde{\rho}_{0}(\mathbf{r}) \rightarrow \tilde{\rho}_{\lambda}(\mathbf{r})=\tilde{\rho}_{0}\left(e^{-\lambda} x, e^{-\lambda} y, e^{2 \lambda} z\right)
$$

In the uniform nuclear matter it is equivalent to the variation of the momentumdistribution $n(\boldsymbol{p})$ as

$$
n_{0}(\boldsymbol{p}) \rightarrow n(\boldsymbol{p})=n_{\lambda}(\boldsymbol{p})=n_{0}\left(\boldsymbol{p}_{\lambda}\right)=n_{0}\left(e^{\lambda} p_{x}, e^{\lambda} p_{y}, e^{-2 \lambda} p_{z}\right)
$$

where $n_{0}(\boldsymbol{p})=\theta\left(p_{F}-|\boldsymbol{p}|\right)$ with the Fermi-momentum $p_{F}$ at the saturation density $\rho_{0}$.

With the variation of the momentum-distribution as $n_{0}(\boldsymbol{p}) \rightarrow n_{0}(\boldsymbol{p})+\delta n_{\boldsymbol{p}}$, the singleparticle energy $\varepsilon(\boldsymbol{p})$ is obtained in the Hartree-Fock framework by

$$
\varepsilon(\boldsymbol{p})=\frac{\delta E_{T}}{\delta n_{\boldsymbol{p}}}=\sqrt{\Pi(p)^{2}+M^{* 2}(p)}+\left.U_{0}(p)\right|_{p_{0}=\varepsilon(\boldsymbol{p})},
$$

¿From this relation we get the following equations 


$$
\begin{gathered}
\left.\frac{\partial E_{T}}{\partial \lambda}\right|_{\lambda=0}=4 \Omega \int \frac{\mathrm{d}^{3} \boldsymbol{p}}{(2 \pi)^{3}}\left\{\left.\frac{\partial n(\boldsymbol{p})}{\partial \lambda}\right|_{\lambda=0}\right\} \varepsilon(\boldsymbol{p})=0 \\
\left.\frac{\partial^{2} E_{T}}{\partial \lambda^{2}}\right|_{\lambda=0}=4 \Omega \int \frac{\mathrm{d}^{3} \boldsymbol{p}}{(2 \pi)^{3}}\left\{\left.\frac{\partial n(\boldsymbol{p})}{\partial \lambda}\right|_{\lambda=0}\right\}\left\{\left.D_{\lambda} \varepsilon(\boldsymbol{p})\right|_{\lambda=0}\right\}
\end{gathered}
$$

The derivative of the single particle energy $\varepsilon$ can be written as

$$
D_{\lambda} \varepsilon\left(\boldsymbol{p}_{\lambda}, \lambda\right)=\frac{\partial \boldsymbol{p}_{\lambda}}{\partial \lambda} \nabla_{\mathbf{p}_{\lambda}} \varepsilon+\frac{\partial \varepsilon}{\partial \lambda}
$$

where the total derivative $\nabla_{\mathbf{p}}$ is defined on the on-mass-shell condition: $p_{0}=\varepsilon(\boldsymbol{p})$. In this equation the second term of the right-hand side $\partial \varepsilon / \partial \lambda$ corresponds to the derivative with the variation of the self-energies at the fixed momentum by changing the momentumdistribution. This term holds the spherical symmetry at the limit of $\lambda \rightarrow 0$, and does not contribute to the integral of the left-hand side in eq.(9).

Substituting eq.(10) into eq.(9), hence, the restoring force of ISGQR $C_{Q}$ becomes

$$
\begin{aligned}
C_{Q} & =\left.\frac{\partial^{2} E_{T} / A}{\partial \lambda^{2}}\right|_{\lambda=0}=-\frac{4 \Omega}{A} \int \frac{\mathrm{d}^{3} \boldsymbol{p}}{(2 \pi)^{3}} \delta\left(|\boldsymbol{p}|-p_{F}\right)\left(\frac{\boldsymbol{p}^{2}-3 p_{z}^{2}}{|\boldsymbol{p}|}\right)\left(\frac{\boldsymbol{p}^{2}-3 p_{z}^{2}}{M_{L}^{*}}\right), \\
& =\frac{12}{5} \frac{p_{F}^{2}}{M_{L}^{*}}
\end{aligned}
$$

where

$$
M_{L}^{*}=\left.\left(2 \frac{d}{d \boldsymbol{p}^{2}} \varepsilon(\boldsymbol{p})\right)^{-1}\right|_{|\boldsymbol{p}|=p_{F}},
$$

which is so called the 'Landau mass' corresponding to the effective mass in the nonrelativistic framework.

In Ref. [5] the mass-parameter of ISGQR is given as

$$
B_{Q}=2 \varepsilon_{F}<\mathbf{r}^{2}>
$$

with the Fermi energy $\varepsilon_{F}$, and then the frequency of ISGQR is obtained as

$$
\omega_{Q}=\sqrt{\frac{C_{Q}}{B_{Q}}}=\sqrt{\frac{6 p_{F}^{2}}{5 M_{L}^{*} \varepsilon_{F}<\mathbf{r}^{2}>}}
$$

This expression is same as that of Ref. [5] with RH except the Landau mass; $M_{L}^{*}=$ $\sqrt{p_{F}^{2}+M^{* 2}}$ in $\mathrm{RH}$.

At the saturation density, the Fermi energy agrees with the total energy per nucleon whose value is almost the same as that of nucleon mass: $\varepsilon_{F}=E_{T} / A \approx M$, In addition, 
nuclear radii are scaled to be proportional to $A^{1 / 3}$ as $<r^{2}>=3 / 5 r_{0}^{2} A^{2 / 3}$ and then we get the frequency $\omega_{Q}$ as

$$
\omega_{Q} \approx \sqrt{\frac{4<T_{K}^{n r}>}{M_{L}^{*} r_{0}^{2}}} A^{-1 / 3},
$$

where $\left\langle T_{K}^{n r}\right\rangle$ is the non-relativistic averaged kinetic energy as

$$
<T_{K}^{n r}>=<\frac{\boldsymbol{p}^{2}}{2 M}>=\frac{3 p_{F}^{2}}{10 M}
$$

This expression completely coincides with that of the non-relativistic model 14

We substitute the empirical experimental values $\omega_{Q} \approx 63 A^{-1 / 3} \mathrm{MeV},<T_{K}^{n r}>\approx$ $25 \mathrm{MeV}$ and $r_{0} \approx 1.125 \mathrm{fm}$ into the eq.(15) and obtain the value of the Landau mass 15 as

$$
M_{L}^{*} / M \approx 0.85
$$

Consequently we cannot find any difference in the expression of ISGQR between the relativistic and non-relativistic frameworks. The main problem is whether we can give the above value of $M_{L}^{*}$ with holding consistency to other observables; the usual analyses indicate that $M^{*} / M=0.55-0.7$, which gives $M_{L}^{*} \approx 0.6-0.75 \mathrm{in} \mathrm{RH}$.

As a next step we explain the details of our calculation in this work. We consider a model of the usual $\sigma-\omega$ model plus the Fock part of the one-pion exchange.

Along this line we define a Lagrangian density in the system as

$$
\begin{aligned}
\mathcal{L}= & \bar{\psi}(i \not \partial-M) \psi+\frac{1}{2} \partial_{\mu} \phi_{a} \partial^{\mu} \phi_{a}-\frac{1}{2} m_{\pi}^{2} \phi_{a} \phi_{a} \\
& +\frac{1}{2} \partial_{\mu} \sigma \partial^{\mu} \sigma-\widetilde{U}[\sigma]-\frac{1}{4} \omega_{\mu \nu} \omega^{\mu \nu}+\frac{1}{2} m_{\omega}^{2} \omega_{\mu} \omega^{\mu}, \\
& +i \frac{f_{\pi}}{m_{\pi}} \bar{\psi} \gamma_{5} \gamma^{\mu} \tau_{a} \psi \partial_{\mu} \phi_{a}+g_{\sigma} \bar{\psi} \psi \sigma-g_{\omega} \bar{\psi} \gamma_{\mu} \psi \omega^{\mu}
\end{aligned}
$$

with

$$
\omega_{\mu \nu}=\partial_{\mu} \omega_{\nu}-\partial_{\nu} \omega_{\mu}
$$

where $\psi, \phi, \sigma$ and $\omega$ are the nucleon, pion, sigma-meson and omega-meson fields, respectively. In the above expression we use the pseudo-vector coupling form as an interaction between Nucleon and pion. The self-energy potential of the $\sigma$-field $\tilde{U}[\sigma]$ is given as

$$
\widetilde{U}[\sigma]=\frac{\frac{1}{2} m_{\sigma}^{2} \sigma^{2}+\frac{1}{3} B_{\sigma} \sigma^{3}+\frac{1}{4} C_{\sigma} \sigma^{4}}{1+\frac{1}{2} A_{\sigma} \sigma^{2}}
$$


The symbols $m_{\pi}, m_{\sigma}$ and $m_{\omega}$ are the masses of $\pi$-, $\sigma$ - and $\omega$-mesons, respectively.

Next we calculate the nucleon self-energies. The nucleon self-energies are separated into the local part and the momentum-dependent part as

$$
U_{\alpha}(p)=U_{\alpha}^{L}+U_{\alpha}^{M D}(p) . \quad(\alpha=s, \mu)
$$

The $\sigma$ - and $\omega$-meson exchange parts produce only very small momentum-dependence of nucleon self-energies [6, [] as their masses are large. In fact the RH and RHF approximations do not give any different results in nuclear matter properties after fitting parameters of $\sigma$ - and $\omega$-exchanges [6]; it is very easily confirmed in the limit of $m_{\alpha} \rightarrow \infty$ at the fixed $g_{\alpha} / m_{\alpha}$. On the other hand the one-pion exchange force is a long range one and makes large momentum-dependence while it does not contribute to the local part in the spinsaturated system. Thus we make the local part by RH of the $\sigma$ - and $\omega$-meson exchanges, and the momentum-dependent part by RHF of the pion exchange. Please note that such a separated method can keep the consistency for the energy-momentum tensor [9].

In this model the local part of the self-energies are given as

$$
\begin{aligned}
U_{s}^{L} & =g_{\sigma}\langle\sigma\rangle \\
U_{\mu}^{L} & =\delta_{0 \mu} \frac{g_{\omega}{ }^{2}}{m_{\omega}^{2}} \rho_{H}
\end{aligned}
$$

where $\langle\sigma\rangle$ is the scalar mean-field obtained as

$$
\frac{\partial}{\partial\langle\sigma\rangle} \tilde{U}[\langle\sigma\rangle]=g_{\sigma} \rho_{s}
$$

In the above equations the scalar density $\rho_{s}$ and the vector Hartree density $\rho_{H}$ are given by

$$
\begin{aligned}
\rho_{s} & =4 \int \frac{\mathrm{d}^{3} \boldsymbol{p}}{(2 \pi)^{3}} n(\boldsymbol{p}) \frac{M_{\alpha}^{*}(p)}{\tilde{\Pi}_{0}(p)}, \\
\rho_{H} & =4 \int \frac{\mathrm{d}^{3} \boldsymbol{p}}{(2 \pi)^{3}} n(\boldsymbol{p}) \frac{\Pi_{0}(p)}{\tilde{\Pi}_{0}(p)},
\end{aligned}
$$

where $n(\boldsymbol{p})$ is the momentum-distribution, and $\tilde{\Pi}_{\mu}(p)$ is defined by

$$
\tilde{\Pi}_{\mu}(p)=\frac{1}{2} \frac{\partial}{\partial p^{\mu}}\left[\Pi^{2}(p)-M^{* 2}(p)\right]
$$

Please note that the Hartree density $\rho_{H}$ is not equivalent to the baryon density $\rho_{B}$ when the self-energies depends on the four momentum. 
As a next step we define the momentum-dependent parts of the self-energies as the Fock parts with the one-pion exchange. When using the pseudo vector (PV) coupling the Fock parts do not become zero at the infinite limit of the momentum $|\boldsymbol{p}|$. One usually erases these contributions by introducing the cut-off parameter. In this work, instead of that, we minus these contributions from the momentum-dependent parts (these contributions can be renormalized into the Hartree parts): $U_{\alpha} \rightarrow U_{\alpha}-U_{\alpha}(p \rightarrow \infty)$. Thus we obtain the momentum-dependent parts of the self-energies as

$$
\begin{aligned}
& U_{s}^{M D}(p)=\frac{3 f_{\pi}^{2}}{2} \int \frac{d^{3} \boldsymbol{k}}{(2 \pi)^{3}} n(\boldsymbol{k}) \frac{M^{*}(k)}{\tilde{\Pi}_{0}(k)} \Delta_{\pi}(p-k), \\
& U_{\mu}^{M D}(p)=-\frac{3 f_{\pi}^{2}}{2} \int \frac{d^{3} \boldsymbol{k}}{(2 \pi)^{3}} n(\boldsymbol{k}) \frac{\Pi_{\mu}(k)}{\tilde{\Pi}_{0}(k)} \Delta_{\pi}(p-k),
\end{aligned}
$$

where the $\Delta_{\pi}(q)$ is the pion propagator defined as

$$
\Delta_{\pi}(q)=\frac{1}{q^{2}-m_{\pi}^{2}}
$$

In the above vector self-energies we omit the tensor-coupling part involving $[\Pi(k)$. $(p-k)](p-k)_{\mu}$. This term is very small if the self-energy is independent of momentum [6], and their momentum-dependence is actually very small as shown later.

Using the above formulation we get the total energy density of the spin-isospin saturated nuclear matter with the momentum-distribution $\left.n_{(} \boldsymbol{p}\right)$ as

$$
\begin{aligned}
E_{T} / \Omega= & 4 \int \frac{\mathrm{d}^{3} \boldsymbol{p}}{(2 \pi)^{3}} n(\boldsymbol{p}) \varepsilon(\boldsymbol{p})+\widetilde{U}[\sigma] \\
& +2 \int \frac{\mathrm{d}^{3} \boldsymbol{p}}{(2 \pi)^{3}} n(\boldsymbol{p}) \frac{M^{*}(p) U_{s}^{M D}(p)-\Pi_{\mu}(p) U^{\mu}(p)}{\tilde{\Pi}_{0}(p)},
\end{aligned}
$$

with the system volume $\Omega$.

Let us show the calculated results in our model using the momentum-dependent Dirac self-energies with the one-pion-exchange. In this calculation we fit the parameters (PF1) for the $\sigma$-and $\omega$ - exchanges to reproduce the saturation properties that the binding energy $B E=16 \mathrm{MeV}$, the incompressibility $K=200 \mathrm{MeV}$ and the effective mass $M^{*} / M=0.7$ at the saturation density $\rho_{0}=0.17 \mathrm{fm}^{-3}$. For comparison we give the results with the momentum-independent self-energies in the parameter-set PM1 [16] which gives the same saturation properties. The detailed values of the parameter-sets PF1 and PM1 are given in Table 1. 
In Fig. 1 we draw the momentum-dependence of the scalar self-energy $U_{s}(p)$ and the time component of the vector self-energy $U_{0}(p)$. It can be seen that the variation of the momentum-dependent self-energies is only $2.5 \%$ at most below Fermi level, which looks very small.

In Fig. 2 we show the density-dependence of the Dirac self-energies $U_{s}$ and $U_{0}$ on the Fermi-surface (a) and the Landau mass (b) with the parameter-sets of PF1 and PM1. Though two results of $U_{s}$ and $U_{0}$ almost agree together, we can see rather large difference in the Landau mass: the value at $\rho_{B}=\rho_{0}$ is $M_{L}^{*} / M=0.85 \mathrm{in} \mathrm{PF} 1$, which is consistent with the value expected by the analysis of ISGQR as shown previously, while the momentumindependent calculation (PM1) gives $M_{L}^{*} / M=0.74$. Hence it is shown that the very small momentum-dependence in the nucleon self-energies enhances the Fermi velocity about 15 $\%$, and gives a significant difference in the Landau mass. Furthermore we can also see an interesting behavior of $M_{L}^{*}$ in PF1, namely, its value agrees with the bare mass at $\rho_{B} \approx 0.5 \rho_{0}$ and becomes larger with the decrease of the density. Effects of small Dirac effective mass are largely canceled at low density by the momentum-dependence created by the one-pion exchange. This fact implies that the non-locality of the self-energies affects nuclear surface properties such as the isovector magnetic moment, whose value is still larger than the Schmidt value [17].

Here we should give a further comment. Bentz et al. have shown in Ref. [18] that the Landau mass is reduced by the one-pion exchange, which is opposite to ours. In this calculation Benz et al. have used the pseudo-scalar (PS) coupling, and the sign of $U_{\mu}^{M D}$ was taken to be opposite to ours.

In order to understand this disagreement more we consider the Schrödinger equivalent potential only with the one-pion exchange. In the non-relativistic limit the Schrödinger equivalent potential is given as

$$
U_{S E P}=-U_{s}+U_{0}+\frac{p^{2}}{2 M} U_{0}
$$

Both the PS and PV couplings make same results up to the first order of $M^{-2}$ and $m_{\pi}^{-2}$. This agreement is caused by the fact $U_{s}^{M D}-U_{0}^{M D} \approx 0$ in the PS coupling at its zeroth order, while the proper PV coupling does not contribute to the zeroth order. However these two parts $U_{s}^{M D}$ and $U_{0}^{M D}$ are not small in the PS coupling because the PS coupling constant $g_{\pi}$ is rather big. In the relativistic mean-field framework, however, 
the self-energies $U_{s}$ and $U_{0}$ have as large strengths as the nucleon mass $M$, and then the cancellation in the non-relativistic limit does not occur. This fact is the origin of the difference between the two kinds of couplings. The full HF calculation with the PS coupling makes too large contribution to the Dirac self-energies [3] while Bentz el al. calculated the Fock term with the perturbative way. Thus a calculation with the PV coupling must be more reliable than that with the PS coupling.

In this paper we have shown that the resonance energy of ISGQR is given with the same formula as the non-relativistic macroscopic theory; this value is determined by the Landau mass $M_{L}^{*}$. The typical value of Dirac effective mass is empirically known as $M^{*} / M=$ $0.55-0.7$, which corresponds to $M_{L}^{*} / M=0.6-0.75$ in the $\mathrm{RH}$ approximation with the momentum-independent Dirac self-energies. Such a small Landau mass overestimates the excited energy of ISGQR. On the contrary, if we introduce the explicit momentumdependences, the Fock part of the one-pion exchange enhances $M_{L}^{*}$. As an example, we have shown that Dirac effective mass $M^{*} / M=0.7$ corresponds to the Landau mass $M_{L}^{*} / M=0.85$, which is consistent with the experimental result of the ISGQR energy, which is in strong contrast to the value. $M_{L}^{*} / M=0.74$ in $\mathrm{RH}$.

Of course we still have some ambiguities in this work. For example the bulk density of finite nuclei is smaller than the saturation density, so that we may discuss the value of the Landau mass at lower density. In Ref. [19], furthermore, it has been reported that the time-dependent mean-field calculations have explained the excited energies of ISGQR for ${ }^{16} \mathrm{O}$ and ${ }^{40} \mathrm{Ca}$. These results are inconsistent with the macroscopic theory. The treated nuclei may be too small or their calculations involve other correlation beyond the macroscopic theory. Thus we should not make a quantitative conclusion on the ISGQR state before investigating it in finite nuclei. Though we still have ambiguities, nevertheless, we can conclude that the momentum-dependence largely affects the Fermivelocity, particularly in the low density region.

Here we should note that although the introduction of the momentum-dependence changes the Landau mass, the depth of self-energies and hence the density dependence of the total energy are affected very little. Thus the approximation to neglect the nonlocality of the Dirac-field should be correct in discussions of many aspects of the nuclear structure in the Dirac approach. However, as for some physical quantities such as Fermi 
velocity, we will have to take account of the non-locality effects in the Dirac approach. This effect cannot be involved even if the density-dependent parameters are introduced into the RH approximation [曰,8].

The authors would like to thank Prof. S. Nishizaki and Dr. Guangjun Mao for stimulating discussions on this work. 


\section{REFERENCES}

[1] B.D. Serot and J. D. Walecka, The relativistic Nuclear Many Body Problem. In J. W. Negele and E. Vogt, editors, Adv.Nucl.Phys.Vol.16, page 1, Plenum Press, 1986, and reference therein.

[2] B. C. Clark, S. Hama, R. L. Mercer, L. Ray and B. D. Serot, Phys. Rev. Lett. 50 (1983) 1644;

S. Hama, B. C. Clark, E. D. Cooper, H. S. Sherif and R. L. Mercer, Phys. Rev. C41, 2737 (1990).

[3] J. A. Tjon and S. J. Wallace, Phys. Rev. C36, 1085 (1987).

[4] D. Hirata, K. Sumiyoshi, B.V. Carlson, H. Toki, Nucl. Phys. A609, 131 (1996).

[5] S. Nishizaki, H. Kurasawa and T. Suzuki, Nucl. Phys. A462, 687 (1987).

[6] C. J. Horowitz and B. D. Serot, Nucl. Phys. A399, 529 (1983).

[7] K. Soutome, T. Maruyama, K. Saito, Nucl. Phys. 507 (1990) 731.

[8] Z.Y. Ma, H.L. Shi and B.Q. Chen, Phys. Rev C50, 3170 (1994); H.L. Shi, B.Q. Chen and Z.Y. Ma, Phys. Rev C52, 144 (1995).

[9] K. Weber, B. Blättel, W. Cassing, H.-C. Dönges, V. Koch, A. Lang and U. Mosel, Nucl. Phys. A539, 713 (1992).

[10] T. Maruyama, B. Blättel, W. Cassing, A. Lang, U. Mosel, K. Weber, Phys. Lett B297, 228 (1992);

T. Maruyama, W. Cassing, U. Mosel, S. Teis and K. Weber, Nucl. Phys A552, 571 (1994).

[11] H. Huber, F. Weber and M. K. Weigel, Phys. Rev. C51, 1790 (1995).

[12] O. Bohigas, A.M. Lane and J. Martorell, Phys. Rep. 51, 267 (1979),

[13] T. Maruyama, Phys. Lett. B245, 325 (1990)

[14] M. Kohno and K. Nado, Prog. Theo. Phys. 61, 1065 (1979).

[15] T. Suzuki, Prog. Theor. Phys. 64, 1627 (1980). 
[16] T. Maruyama, H. Fujii, T. Muto and T. Tatsumi, Phys. Lett. B337, 19 (1994);

H. Fujii, T. Maruyama, T. Muto and T. Tatsumi, Nucl. Phys. A597, 645 (1996).

[17] A. Bouyssy, S. Marcos and J.F. Mathiot, Nucl. Phys.A415, 497 (1984).

[18] W. Benz, A. Arima and H. Baier, Nucl. Phys. A541, 333 (1992).

[19] D. Vretenar, H. Berghammer and P. Ring, Nucl. Phys. A581 (1995) 679. 


\begin{tabular}{|c|ccccc|}
\hline \hline & $g_{\sigma}$ & $g_{\omega}$ & $B_{\sigma}$ & $A_{\sigma}$ & $f_{\pi}$ \\
\hline PF1 & 9.699 & 9.880 & 27.61 & 6.134 & 1.008 \\
\hline PM1 & 9.408 & 9.993 & 23.52 & 5.651 & 0.0 \\
\hline
\end{tabular}

Parameter sets in this paper. In all cases have used $m_{\pi}=138$

$\mathrm{MeV}, m_{\sigma}=550 \mathrm{MeV}, m_{\omega}=783 \mathrm{MeV}$ and $C_{\sigma}=0$. 


\section{Figure Captions}

Fig. 1 Momentum-Dependence of the scalar (a) and vector (b) self-energies. The solid and dashed lines indicate the results in PF1 and PM1, respectively. The dotted line denotes the position of the Fermi momentum at $\rho_{B}=\rho_{0}$.

Fig. 2 Density-dependence of the Dirac self-energies $U_{s}$ and $U_{0}$ on the Fermi-surface (a) and the Landau mass (b) the Landau mass (b). The solid and dashed lines indicate the results for PF1 and PM1, respectively, and the full square in (b) denotes the value expected empirically from ISGQR. 
Dirac Potential

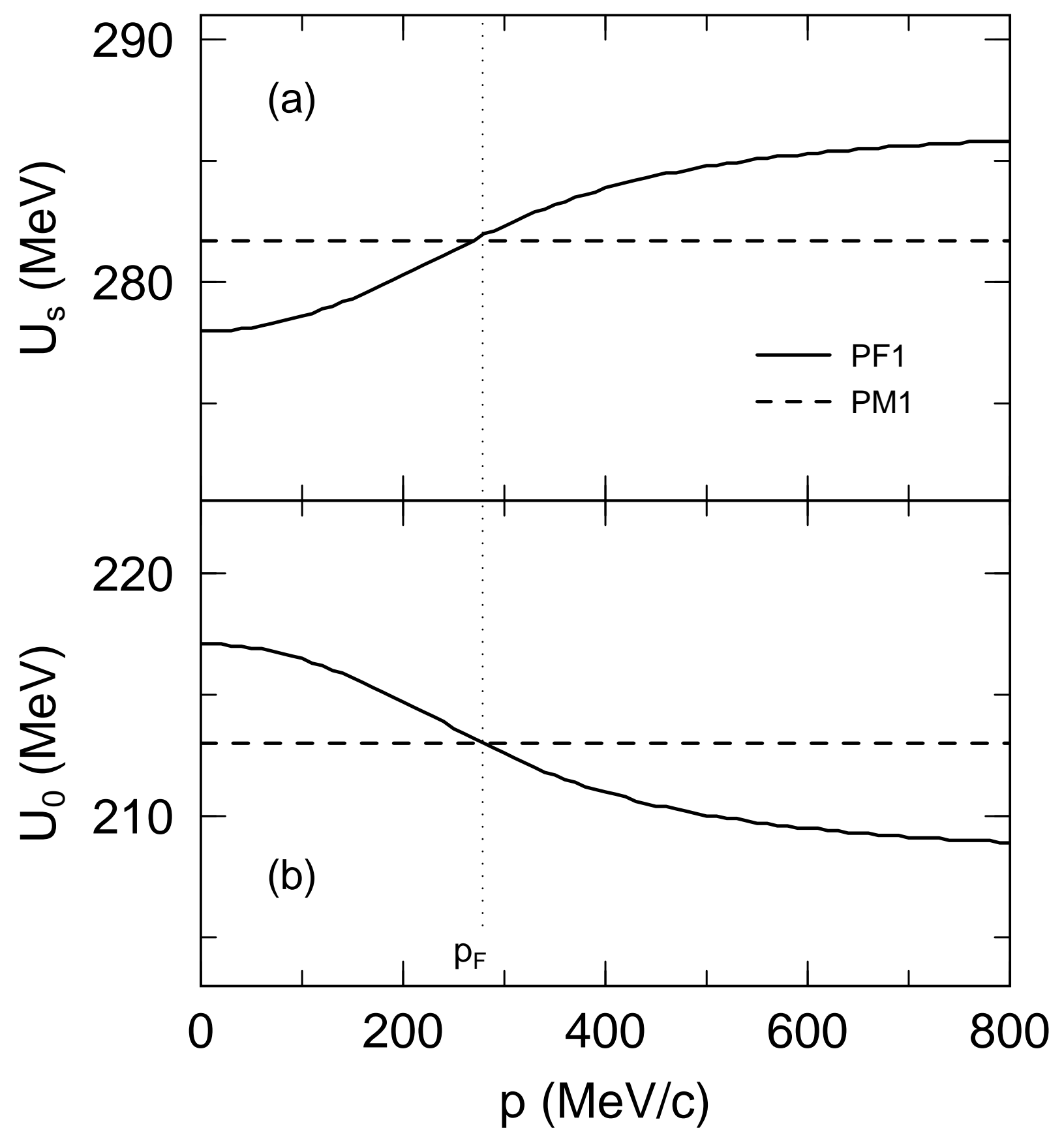




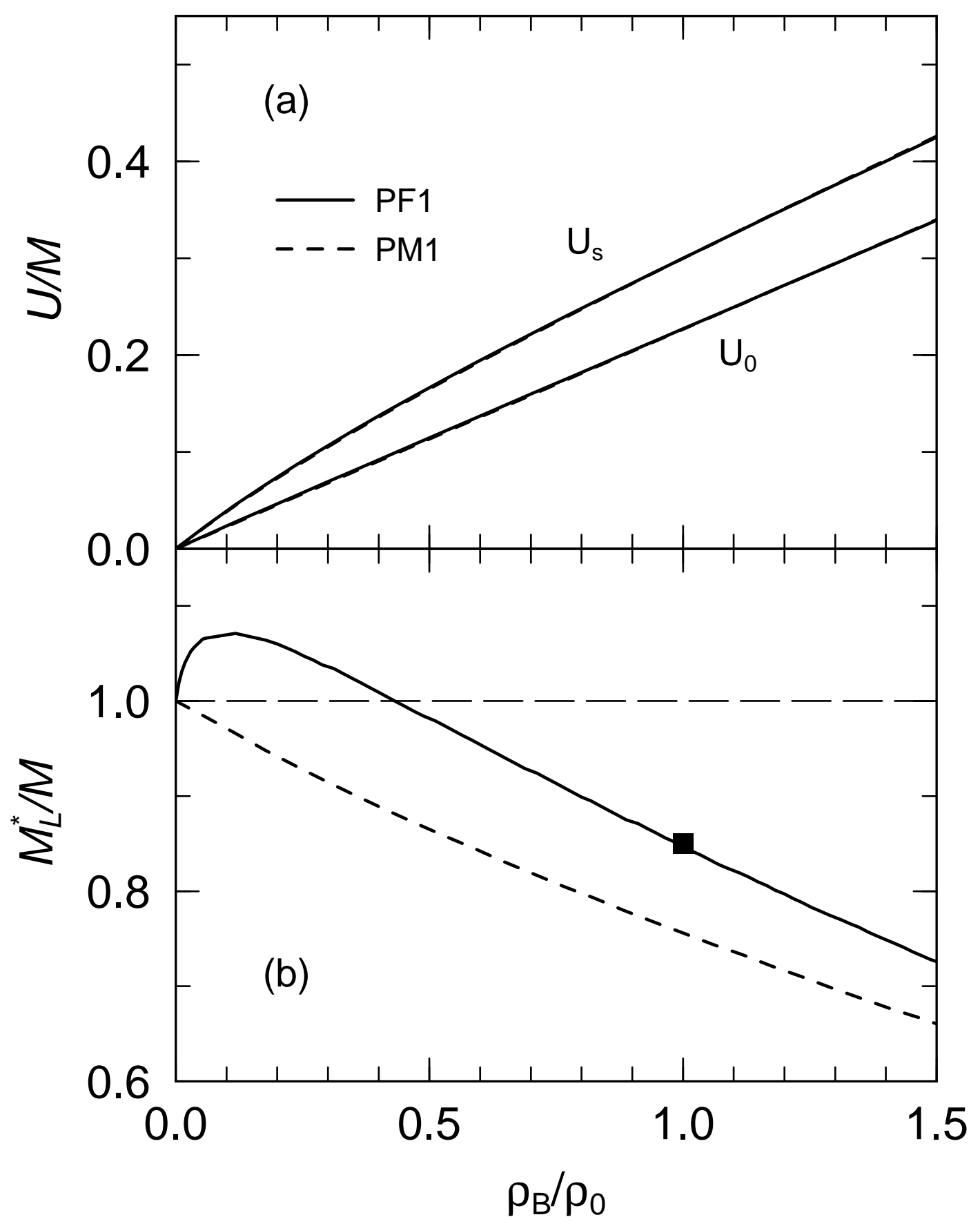

